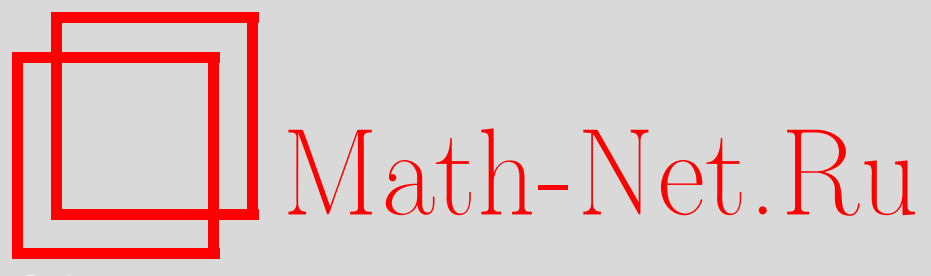

В. И. Ким, И. Б. Кожухов, О полугруппах изотонных преобразований частично упорядоченных множеств, УМН, 2007, том 62, выпуск 5, 155-156

DOI: https://doi.org/10.4213/rm8526

Использование Общероссийского математического портала Math-Net.Ru подразумевает, что вы прочитали и согласны с пользовательским соглашением http://www . mathnet.ru/rus/agreement

Параметры загрузки:

IP : 35.173 .137 .237

26 апреля 2023 г., 02:24:09

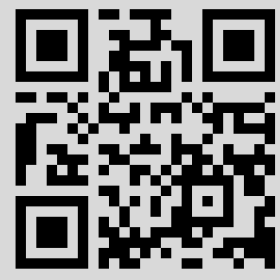




\title{
О полугруппах изотонных преобразований частично упорядоченных множеств
}

\author{
В. И. Ким, И. Б. Кожухов
}

Обозначим через $T(X)$ полугруппу всех преобразований множества $X$, т. е. отображений $\alpha: X \rightarrow X$ с умножением, определенным по формуле $x(\alpha \beta)=(x \alpha) \beta$. Алгебраическое строение полугруппы $T(X)$ хорошо известно (см., например, [1]). Полугруппа $T(X)$ регулярна, т. е. для любого $\alpha \in T(X)$ существует $\beta \in T(X)$ такое, что $\alpha \beta \alpha=\alpha$. Если $X-$ множество, наделенное некоторой структурой, то естественно рассматривать такие преобразования, которые согласованы с этой структурой. Например, если $X$ - топологическое пространство, то интерес представляет полугруппа $C(X)$ всех непрерывных отображений $X \rightarrow X$ (см. обзор [2]). Если $X$ - универсальная алгебра, то полугруппа всех преобразований $X \rightarrow X$, сохраняющих операции, есть полугруппа эндоморфизмов End $X$, играющая важную роль в общей алгебре. Если $X-$ частично упорядоченное множество, то пусть $O(X)$ обозначает полугруппу всех преобразований $\alpha: X \rightarrow X$ множества $X$, сохраняющих порядок, т. е. $x \leqslant y \Rightarrow x \alpha \leqslant y \alpha$ для всех $x, y \in X$. Назовем такие преобразования изотонными. Различные вопросы, связанные с полугруппами изотонных преобразований, были освещены в обзоре [3]. Полугруппа $O(X)$ содержит большую информацию о частично упорядоченном множестве $X$. Л. М. Глускиным было доказано (см. [4]), что изоморфизм $O(X) \cong O(Y)$ полугрупп преобразований двух частично упорядоченных множеств $X$ и $Y$ (даже квазиупорядоченных) влечет, что $X$ изоморфно или антиизоморфно $Y$.

Полугруппа $O(X)$ регулярна далеко не всегда. Прежде чем формулировать условия регулярности этой полугруппы, введем некоторые определения и обозначения. Цепь - линейно упорядоченное множество; антицепь - частично упорядоченное множество $X$ такое, что $x \nless y$ при любых $x, y \in X$. Пусть $Y$ - произвольное непустое множество, $x_{0}$ и $z_{0}$-любые элементы, не принадлежащие $Y$. Положим $L_{Y}=\left\{x_{0}, z_{0}\right\} \cup Y$ и определим порядок на $L_{Y}$ следующим образом: $x_{0}<y<z_{0}$ при всех $y \in Y$ (остальные пары элементов не связаны отношением “ $<$ "). Далее, пусть $Y$ и $Z$ - произвольные непустые множества и $Y \cap Z=\varnothing$. Положим $F_{Y, Z}=Y \cup Z$ с введенным на этом множестве порядком $a<b \Leftrightarrow a \in Y \& b \in Z$. Для непустых непересекающихся множеств $Y, Z$ с выделенными в них элементами $y_{0} \in Y, z_{0} \in Z$ положим $G_{Y, Z}=Y \cup Z$ с введенным на этом множестве порядком $a<b \Leftrightarrow\left(a=y_{0} \& b \in Z\right) \vee\left(a \in Y \& b=z_{0}\right)$. Наконец, пусть $C_{6}=\{1,2,3,4,5,6\}$ с порядком $1<4,6,2<4,5,3<5,6$. Следующая теорема, принадлежащая А.Я. Айзенштат, дает условия регулярности полугруппы $O(X)$ для широкого класса частично упорядоченных множеств.

Теорема 1 [5]. Пусть $X$ - частично упорядоченное множество, не являющееся цепью. Тогда полугруппа $O(X)$ регулярна в том и толъко том случае, если $X$ антицепь или $X$ изоморфно одному из множеств $L_{Y}, F_{Y, Z}, G_{Y, Z}, C_{6}$.

Условия регулярности полугруппы $O(X)$ в случае, когда $X$ - цепь, были даны в [5] в терминах некоторого бинарного отношения, определенного на множестве пар $\left(X_{1}, \alpha\right)$, где $X_{1} \subseteq X$, а $\alpha$ - изотонное вложение $X_{1}$ в $X$. Мы получили для счетных цепей $X$ более прозрачные условия регулярности полугруппы $O(X)$. Формулировку результатов приведем после некоторых определений.

Пусть $\Gamma$ - цепь (не обязательно счетная). Сечением $(A, B)$ называется разбиение $\Gamma=A \cup B$ цепи $\Gamma$ на два непустых подмножества $A$ и $B$ таких, что $a<b$ при всех $a \in A$, $b \in B$. Сечение $(A, B)$ назовем дырой, если множество $A$ не имеет точной верхней грани (а значит, множество $B$ не имеет точной нижней грани). Цепь называется полной, если никакое ее сечение не является дырой. Для элемента $x \in \Gamma$ полагаем $x^{\Delta}=\{y \mid y \leqslant x\}, x^{\nabla}=\{y \mid y \geqslant x\}$.

Теорема 2 [6]. Пусть Г - счетная цепь. Тогда полугруппа $O(\Gamma)$ регулярна в том и только том случае, если выполнено одно из следующих условий: 1) Г - полная цепь, 
имеющая минимальный и максимальный элементь; 2) Г не имеет максимального, но имеет минимальный элемент и для любого $x \in \Gamma$ множество $x^{\Delta}$ удовлетворяет условию максимальности; 3) Г не имеет минимального, но имеет максимальный элемент и для любого $x \in \Gamma$ множество $x^{\nabla}$ удовлетворяет условию минимальности; 4) $\Gamma \cong \mathbb{Z}$; 5) $\Gamma$ имеет минимальный и максимальный элементы, $\Gamma=A \cup B$ $u(A, B)$ - дыра, причем для любого $x \in A$ множество $x^{\Delta}$ удовлетворяет условию максимальности и для любого $x \in B$ множество $x^{\nabla}$ удовлетворяет условию минимальности.

СлЕДствиЕ. Пусть Г - счетная цепь, причем полугруппа $O(Г)$ регулярна. Если Г имеет дыру, то эта дыра единственна.

Назовем элемент $а$ полугруппы $S$ слабо регулярным справа, если $a \in a S a S$, и слабо регулярным слева, если $a \in S a S a$. Полугруппа называется слабо регулярной справа $($ слева), если все ее элементы слабо регулярны справа (слева). Рассмотрение главных факторов полугрупп (см. [1]) показывает, что в конечных полугруппах понятия регулярного $(a \in a S a)$ и слабо регулярного слева (справа) элемента совпадают. Естественно поставить вопрос об условиях слабой регулярности полугруппы изотонных преобразований частично упорядоченного множества. Оказывается, что ряд утверждений, на основе которых доказывается теорема 1, обобщается с регулярных на слабо регулярные полугруппы, как показывает приводимое ниже предложение. Частично упорядоченное множество $X$ назовем свлзным, если наименьшее отношение эквивалентности на $X$, содержащее отношение $\leqslant$, совпадает с $X \times X$. Это равносильно тому, что граф отношения $\leqslant$ на $X$ связен.

ПреДЛОЖЕНИЕ. 1) Пусть $X$ - частично упорлдоченное множество. Если полугруппа $O(X)$ слабо регулярна слева или справа, то либо $X$ связно, либо $X$ - античепъ. 2) Если $X$ - связное частично упорядоченное множество и полугруппа $O(X)$ слабо регулярна слева или справа, то каждая цепь в $X$ содержит не более трех элементов.

Утверждение 1) в этом предложении остается верным, если условие слабой регулярности полугруппы $O(X)$ заменить следующим условием: "каждый элемент полугруппы $O(X)$ слабо регулярен слева или справа".

Отображение $\alpha \in T(X)$ назовем конечным, если $|X \alpha|<\infty$, и коконечным, если $|X \backslash X \alpha|<\infty$. Множество $O_{\text {fin }}(X)$ всех конечных и множество $O_{\text {cofin }}(X)$ всех коконечных изотонных преобразований множества $X$ - полугруппы, являющиеся подполугруппами полугруппы $O(X)$. Имеет место

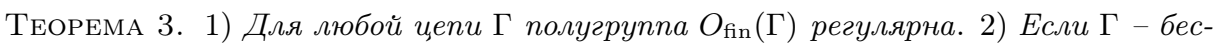
конечная счетная цепь, то полугруппа $O_{\mathrm{cofin}}(\Gamma)$ не регулярна.

\section{Список литературы}

[1] А. Клиффорд, Г. Престон, Алгебрачческая теория полугрупп, Т. 2, Мир, М., 1972. [2] K. D. Magill, Jr., Semigroup Forum, 11:1 (1976), 189-282. [3] V. A. Molchanov, Semigroup Forum, 27:1 (1983), 155-199. [4] Л.М.Глускин, УМН, 16:5 (1961), 157-162. [5] А. Я. Айзенштат, Уч. Записки Ленингр. гос. пед. ин-та им. А. И. Герцена, 387 (1968), 3-11. [6] В. И. Ким, И. Б. Кожухов, Фундам. и прикл. матем., 12:8 (2006), 97-104.

\section{В. И. Ким (V. I. Kim)}

Московский государственный институт электронной техники (технический университет)

E-mail: victor-kim@mail.ru

\section{И. Б. Кожухов (I. В. Kozhukhov)}

Московский государственный институт электронной техники (технический университет)

E-mail: kozhuhov_i_b@mail.ru
Представлено А. В. Михалёвым Принято редколлегией 01.08.2007 